\title{
CATAMENIAL PNEUMOTHORAX: A MULTIFACTORIAL ETIOLOGY
}

Peter Fonseca, MD, PhD, Bethesda, Md

The physiologic mechanism of catamenial pneumothorax remains obscure. A clear understanding of the etiology has been lacking since the first description by Maurer, Schaal, and Mendez ${ }^{1}$ in 1958 of recurrent spontaneous pneumothorax associated with the onset of menstruation. A case is presented with operative findings and a postoperative course that shed some light on this entity. The importance of alveolar rupture, sensitized by hormonal changes as part of a multifactorial milieu, is described.

Clinical summary. A 39-year old woman had 6 documented right spontaneous pneumothoraces during a 2-year period, all within 5 to 7 days of menses. There were no occurrences during a 6-month trial of danazol.

The patient's previous medical history was significant for intra-abdominal endometriosis diagnosed by laparoscopy, which was part of an extensive infertility workup (no pneumothorax at that time). Results of physical examination were unremarkable, other than that she was a tall, thin woman. Her health at examination was excellent. She was an active duty military officer and an avid runner.

The patient sought treatment with right chest pain and a $40 \%$ right pneumothorax. The recurrent, cyclic nature of her pneumothoraces in association with menses suggested catamenial pneumothorax, and we performed a right thoracoscopy. The lung was entirely normal in appearance, other than an apical adhesion that was taken down with electrocautery. No blebs, bullae, or endometrial implants were found within the parenchyma.

The diaphragm had multiple fenestrations in its central portion (Fig 1) and the pericardial fat had numerous hemosiderin-laden macrophages, suggesting previous bleeding from a nearby source. The diaphragm was oversewn and the parietal pleura was mechanically abraded to complete the procedure. Diaphragmatic biopsy samples from these fenestration and pericardial implants showed no evidence of endometriosis.

Three months after the operation the patient had a recurrent right pneumothorax related to menses during a 6-mile run. A

From the Department of Cardiothoracic Surgery, National Naval Medical Center, Bethesda, Md.

The views expressed in this article are those of the author and do not reflect the official policy or position of the Department of the Navy, Department of Defense, or the US Government.

Received for publication June 9, 1998; accepted for publication June 16, 1998.

Address for reprints: Peter Fonseca, MD, PhD, Department of Cardiothoracic Surgery, National Naval Medical Center, 8901 Wisconsin Ave, Bethesda, MD 20889.

J Thorac Cardiovasc Surg 1998;116:872-3

12/54/92609

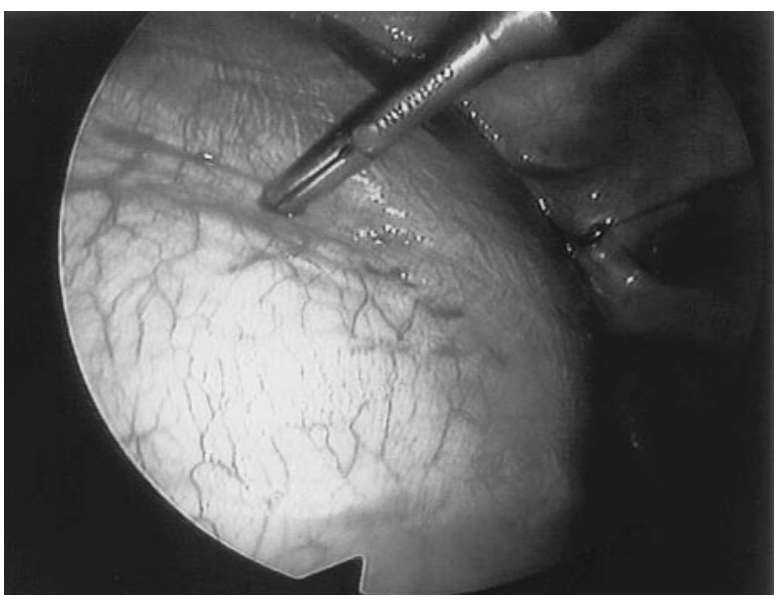

Fig 1. Thoracoscopic view of the central tendon of the right diaphragm demonstrating a probe through a fenestration.

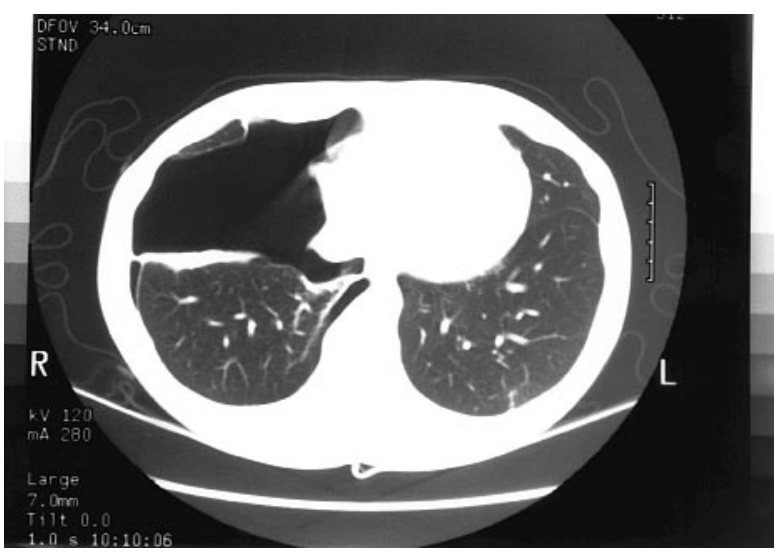

Fig 2. Computed tomographic scan of the chest demonstrating middle lobe collapse after initial thoracoscopic treatment of pneumothorax.

computed tomographic scan confirmed a collapse of the middle lobe (Fig 2). Repeated thoracoscopy showed no evidence of diaphragmatic fenestration, endometriosis, or blebs, and a talc pleurodesis was performed. No further episodes of pneumothorax have occurred in the ensuing 2 years.

Discussion. Three proposed mechanisms for the cyclic occurrence of pneumothorax in women have been described in the literature. This case represents some features of all 3 hypotheses. The 3 proposals are as follows. (1) In the 
anatomic model, ${ }^{1,2}$ during menstruation there is an open connection between the atmosphere and the peritoneal cavity because the cervical mucus plug is absent. Air can migrate through the tube into the abdominal cavity and through diaphragmatic fenestrations into the chest to cause pneumothorax. (2) In the metastatic model,, 34 similar to air migration, endometrial implants find their way through fenestrations or through transdiaphragmatic lymphatic channels to the surface of the chest and lung or are carried by the blood to the lung parenchyma. (3) In the physiologic hypothesis, ${ }^{5}$ high serum levels of prostaglandins in menstruating women may cause vasospasm and bronchospasm with possible rupture of alveoli, which results in pneumothorax. Blebs or bullae might also be more susceptible to rupture through hormonal changes.

Criticisms of the anatomic hypothesis (congenital fenestration) include failure to explain why pneumothorax is not seen in a large number of patients undergoing celioscopy. Many patients are presumed to have congenital fenestrations of the diaphragm. ${ }^{6}$ Indeed, our patient underwent laparoscopy several years earlier without incident. In addition, Soderberg and Dahlquist ${ }^{7}$ noted the persistence of recurrent pneumothoraces in 8 patients when hysterectomies had been performed, and diaphragmatic fenestrations have only been found in between $19 \%$ and $23 \%$ of reported catamenial cases. ${ }^{8}$

Support for the metastatic theory is also less than universal. In a literature review, Schoenfield ${ }^{8}$ noted that pelvic endometriosis was demonstrated in only $22 \%$ to $37 \%$ of cases and pleural, and diaphragmatic endometrial implants were seen in between $23 \%$ and $35 \%$ of literature reports.

The physiologic hypothesis seems attractive because when intrathoracic pathology is found (fenestration, implants, blebs) and addressed, further recurrence may be seen if pleurodesis is not performed. ${ }^{2,5}$ This indicates the presence of a recurrent, cyclic, and possibly de novo stimulus with resultant outcome. In our case the initial thoracoscopy showed evidence of an apical adhesion, perhaps old endometrial implants (hemosiderin-laden macrophages), and diaphragmatic fenestrations. At postoperative recurrence, however, none of these were apparent. The middle lobe was the lobe least in contact with nonabraded surfaces (diaphragm and pericardium) and therefore had the least adhesion.

The pneumothorax occurred during menses and physical stress (running). The patient demonstrated no evidence of either endometrial implantation, blebs, or fenestrations as the causal factor. It is believed that the fenestrations found at the first thoracoscopy were an incidental finding (none identified at the second operation and no pneumothorax caused during insufflation of earlier laparoscopy). As such, alveolar rupture seems the likely cause.

A physiologic stimulus resulting in an anatomic lesion in an environment of cyclic change may cause this unique type of pneumothorax. Although all 3 hypotheses could explain findings in the case presented here, only the physiologic etiology best explains the recurrent pneumothorax after the first procedure. After suppression of the pituitary ovarian axis with danazol, the physiologic environment for alveolar rupture was no longer present.

Conclusion. We conclude that an alveolar rupture related to hormonal changes associated with menses was the principle cause of pneumothorax in this patient. The combination of elevated prostaglandin levels at the time of menses, which result in bronchiolar constriction and alveolar rupture, with respiratory exertion resulted in pneumothorax.

\section{REFERENCES}

1. Maurer ER, Schaal JA, Mendez FL. Chronic recurring spontaneous pneumothorax due to endometriosis of the diaphragm. JAMA 1958;168;2013-4.

2. Crutcher RR, Waltuch TL, Blue ME. Recurrent spontaneous pneumothorax associated with menstruation. J Thorac Cardiovasc Surg 1967;54:599-602.

3. Shiraishi T. Catamenial pneumothorax: report of a case and review of the Japanese and non-Japanese literature. Thorac Cardiovasc Surg 1991;33:304-7.

4. Van Schil PE, Vercauteren SR, Vermeire PA, Nackaerts YH, Van Marck EA. Catamenial pneumothorax caused by thoracic endometriosis. Ann Thorac Surg 1996;62:585-6.

5. Rossi NP, Goplerud CP. Recurrent catamenial pneumothorax. Arch Surg 1974;109:173-6.

6. Carter EJ, Ettensohn DB. Catamenial pneumothorax. Chest 1990;98:713-6.

7. Soderberg $\mathrm{CH}$, Dahlquist EH. Catamenial pneumothorax. Surgery 1976;79:236-8.

8. Schoenfeld A, Ziv E, Zeelel Y, Ovadia J. Catamenial pneumothorax: a literature review and report of an unusual case. Obstet Gynecol Surv 1986;41:20-4. 